\title{
Aquatic Therapy Versus Conventional Land-Based Therapy for Parkinson's Disease: An Open-Label Pilot Study
}

\author{
Jamile Vivas, PhD, PT, Pablo Arias, PhD, Javier Cudeiro, PhD, MD
}

\begin{abstract}
Objectives: To assess and compare 2 different protocols of physiotherapy (land or water therapy) for people with Parkinson's disease (PD) focused on postural stability and self-movement, and to provide methodological information regarding progression within the program for a future larger trial.

Design: Randomized, controlled, open-label pilot trial. Setting: Outpatients, Parkinson's disease Center of Ferrol - Galicia (Spain). Participants: Individuals $(\mathrm{N}=11)$ with idiopathic PD in stages 2 or 3 according to the Hoehn and Yahr Scale completed the investigation (intervention period plus follow-up).

Interventions: After baseline evaluations, participants were randomly assigned to a land-based therapy (active control group) or a water-based therapy (experimental group). Participants underwent individual sessions for 4 weeks, twice a week, for 45 minutes per session. Both interventions were matched in terms of exercise features, which were structured in stages with clear objectives and progression criteria to pass to the next phase.

Main Outcome Measures: Participants underwent a first baseline assessment, a posttest immediately after 4 weeks of intervention, and a follow-up assessment after 17 days. Evaluations were performed OFF-dose after withholding medication for 12 hours. Functional assessments included the Functional Reach Test (FRT), the Berg Balance Scale (BBS), the UPDRS, the 5-m walk test, and the Timed Up and Go test.

Results: A main effect of both therapies was seen for the FRT. Only the aquatic therapy group improved in the BBS and the UPDRS.

Conclusions: In this pilot study, physiotherapy protocols produced improvement in postural stability in PD that was significantly larger after aquatic therapy. The intervention protocols are shown to be feasible and seem to be of value in amelioration of postural stability-related impairments in PD. Some of the methodological aspects detailed here can be used to design larger controlled trials.
\end{abstract}

Key Words: Hydrotherapy; Parkinson disease; Physical therapy modalities; Rehabilitation.

PHYSICAL THERAPY (physiotherapy) is one of the most conventional therapies in Parkinson's disease (PD). ${ }^{1-3}$ Despite this, the role of physiotherapy in objectively decreasing the severity of signs of the disease has not been fully addressed. Kwakkel et $\mathrm{al}^{4}$ critically reviewed work assessing the impact of physiotherapy on PD, concluding that methodological pitfalls are common in most published work. Deane et $\mathrm{al}^{5}$ concluded that most of the studies about paramedical therapies show limited evidence about efficacy, principally because of the great variability of procedures and the lack of common criteria. Different land-based physiotherapy protocols have promoted improvements on gait parameters, such as velocity $1,{ }^{6-9}$ and step amplitude, ${ }^{10}$ but it is difficult to extract general methodological guidelines, which are necessary to improve intervention procedures and to set a solid framework on which to establish proper scientific discussion. Aquatic physiotherapy can also be an alternative to land-based protocols. Different authors have suggested this kind of therapy as a means to improve the quality of life, reduce postural instability and the risk of falling in the elderly, ${ }^{11}$ and to enhance treatments for different disorders (eg, women with osteoporosis ${ }^{12,13}$ or lower extremity arthritis ${ }^{14}$ ). Aquatic therapy is also indicated for the treatment of neurologic disorders such as vestibular dysfunction ${ }^{15}$ or cerebral palsy ${ }^{16}$ It has been proposed that the utilization of an aquatic environment can promote significant therapeutic results such as (1) a 
decrease in muscle tone ${ }^{17,18}$; (2) an improvement of postural stability ${ }^{19}$; (3) an increment of functional mobility $^{20}$; and (4) a reduction of spasm severity in spasticity. ${ }^{21}$

Aquatic therapy is currently used as a treatment for individuals with PD in clinical practice, but to our knowledge there is no specific and controlled research focused on an aquatic physiotherapy intervention for PD. It is important to reiterate that for both aquatic- and land-based protocols, there is a lack of available rigorous methodological protocols, for instance, stating different protocol phases with clear objectives, procedures, and progression criteria.

This study addresses the need and importance of validating the efficacy of standardized protocols when applied to the PD population in different yet complementary therapeutic settings and environments. The aim of this pilot study was 3-fold: (1) to apply 2 different protocols of physiotherapy (land and water therapy) focused on postural stability, maintaining body position, transferring oneself, and changing body positions; (2) to assess the feasibility of the programs for a future larger trial; and (3) to provide a methodological proposal, based on progression criteria within the program, as a means to develop a larger trial.

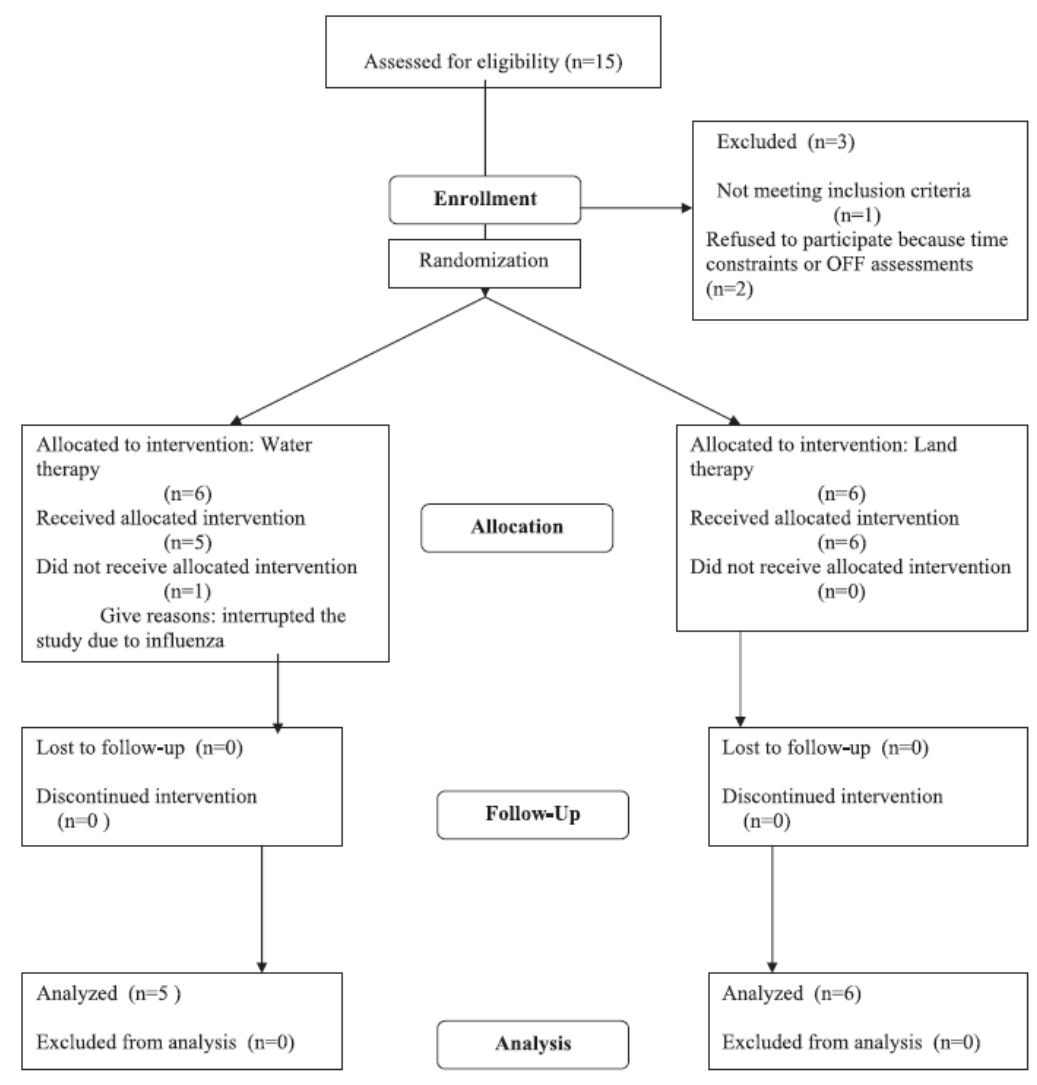

Fig 1. Trial flowchart.

\section{METHODS}

\section{Participants}

Fifteen individuals with idiopathic PD from the Parkinson's Disease Association of Ferrol, Galicia, were assessed for eligibility to participate in our study (fig 1). Twelve participants (8 men, 4 women; mean age \pm SD, $67 \pm 5.5 \mathrm{y}$ ) were initially enrolled (table 1 ), and 11 participants successfully completed the whole program. The inclusion criteria were as follows: ability to follow a stable medication schedule; to be in PD stages 2 or 3 according to the Hoehn and Yahr Scale while in the OFF-medication phase (in absence of the effect of medication); and lack of dementia (Mini-Mental State Examination score, $\geq 24$ ). Participants were excluded if they were unable to walk independently or had undergone surgical treatment for PD. The procedures conformed to the Helsinki Declaration and were approved by our institutional ethics committee. Participants signed appropriate consent forms. 
Table 1: Characteristics of Participants With PD

\begin{tabular}{|c|c|c|c|c|c|c|c|}
\hline Patient (Group) & Sex & $\begin{array}{c}\text { Dominant } \\
\text { Hand }\end{array}$ & $\begin{array}{l}\text { More Affected } \\
\text { Side } \\
\end{array}$ & Age (y) & $\begin{array}{c}\text { Time Since } \\
\text { Diagnosis }\end{array}$ & MMSE & H \& Y \\
\hline 1 (Control) & M & $\mathrm{R}$ & $\mathrm{R}$ & 68 & 7 & 29 & 2 \\
\hline 2 (Control) & $\mathrm{F}$ & $\mathrm{R}$ & $\mathrm{R}$ & 73 & 13 & 24 & 2 \\
\hline 3 (Control) & $\mathrm{F}$ & $\mathrm{R}$ & $\mathrm{R}$ & 57 & 11 & 28 & 2.5 \\
\hline 4 (Control) & M & $\mathrm{R}$ & $\mathrm{L}$ & 77 & 9 & 26 & 3 \\
\hline Group mean \pm SD & NA & NA & NA & $68.33 \pm 6.92$ & $7.83 \pm 3.92$ & $27.5 \pm 2.17$ & $2.4 \pm .55$ \\
\hline 7 (Experimental) & $\mathrm{M}$ & $\mathrm{R}$ & $\mathrm{R}$ & 63 & 3 & 25 & 3 \\
\hline 8 (Experimental) & $\mathrm{F}$ & $\mathrm{R}$ & $\mathrm{R}$ & 68 & 7 & 29 & 2.5 \\
\hline 9 (Experimental) & $\mathrm{F}$ & $\mathrm{R}$ & $\mathrm{R}$ & 63 & 5 & 29 & 2 \\
\hline 10 (Experimental) & $\mathrm{F}$ & $\mathrm{R}$ & $\mathrm{L}$ & 65 & 4 & 30 & 3 \\
\hline 11 (Experimental) & M & $\mathrm{R}$ & $\mathrm{R}$ & 72 & 3 & 25 & 2.5 \\
\hline
\end{tabular}

Abbreviations: F, female; H \& Y, Hoehn and Yahr Scale; L, left; M, male; MMSE, Mini-Mental State Examination; NA, not applicable; R, right.

\section{Procedure}

Participants underwent the following sequence of events; a first baseline assessment (Pretest); 4 weeks of intervention during ON-periods (in presence of the effect of medication); retest after intervention (Posttest); and a follow-up assessment after 17 days (Posttest-2) (table 2). The evaluations were conducted at the facilities of the patient's association. For each participant, all evaluations were performed under the same conditions throughout the whole program. Evaluations were performed OFFdose, after withholding medication for 12 hours. ${ }^{22-24}$

Aquatic intervention took place in a city spa. The pool was $3.55 \mathrm{~m}$ wide, $7.75 \mathrm{~m}$ long, and $1.30 \mathrm{~m}$ deep throughout it, and the water temperature was about, but not less than, $32^{\circ} \mathrm{C}\left(89.6^{\circ} \mathrm{F}\right)$.

\section{Evaluations}

Given that therapy was focused on postural stability (a cardinal sign of the disease related to gait impairment), transferring oneself (International Classification of Functioning, Disability and Health code d420), and changing body positions (International Classification of Functioning, Disability and Health code d410); the main tests used to evaluate effectiveness were the following:

1. Functional Reach Test (FRT): A Harpender anthropometer, a calibrated to the nearest millimeter and supported by a tripod, was horizontally positioned at shoulder height. Participants were asked to reach as forward as possible, in a parallel plane with the measuring device, ${ }^{25}$ while maintaining a fixed base of support. The distance between the arms' initial position and the maximal forward reach was defined as the functional reach value. Participants performed this task 3 times for each arm.

2. Berg Balance Scale (BBS): Briefly, the BBS is a 14-item scale widely used for assessing balance. The items are scored from 0 (unable to execute the task) to 4 (independent) on the basis of the ability to complete a task, with the higher score indicating the degree of independence displayed while performing the tasks. ${ }^{26}$

3. Gait: Participants had to walk along a 5-m walkway (3 times) using their normal, preferred walking pattern, turn around a pivot 3 (U-turn), and return to their starting position. Photocells were placed at the beginning and at the end of the corridor (.58m before pivot point), and the test was recorded by a video camera. ${ }^{\text {b }}$

4. Timed Up and Go (TUG): Briefly, for the TUG test, participants were seated on a chair, and once commanded, they had to stand up, walk at their preferred walking pattern for $3 \mathrm{~m}$, turn, come back, and sit down on the chair again. ${ }^{27}$ The time taken was recorded with a stopwatch. Participants performed this task 3 times.

5. Unified Parkinson's Disease Rating Scale (UPDRS): A standard score was calculated. 


\section{Interventions}

After baseline evaluation (Pretest), participants were randomly assigned to either a land-based therapy (active control group) or a water-based therapy (experimental group). A single physiotherapist performed the sessions with each participant individually for all sessions over the 4 weeks. The 45 -minute sessions were conducted twice a week on nonconsecutive days.

\section{Therapy Protocols}

Both land- and water-based protocols consisted of 4 sections of exercises, chiefly oriented to different body structures appropriate to movement (International Classification of Functioning, Disability and Health code): trunk (s760), pelvis (s750), lower extremity (s750), and upper extremity (s730) including shoulder region (s720). Domains focused on were (1) warm-up exercises, (2) trunk mobility exercises, (3) postural stability (b715), and (4) transferring oneself (d420) and changing body positions (d410). Many of the exercises followed the Halliwick method28 (for an excellent depiction of them, including video support, see Brody and Geigle29).

Participants within the program were encouraged to progress, based on stated progression criteria and progression exercises. Progression criteria were abilities to be achieved through a set of exercises, such that when those abilities were achieved, more complex exercises (progression exercises) were introduced, oriented toward achieving the next progression criteria, more demanding than the first (tables 3 and 4). Progression criteria were as follows:

Table 2: Study Design

\begin{tabular}{llllll}
\hline PROCEDURE & PREINTERVENTION & Active Control Group & POSTINTERVENTION & Follow-up & 2nd \\
& ASSESSMENT & LAND-BASED & ASSESSMENT & 17d & POSTINTERVENTION \\
& (Pretest) & PROGRAM (4wk) & (Posttest) & without & ASSESSMENT \\
& & & therapy & (Posttest-2) \\
& & & \\
& & & \\
& Experimental Group & & \\
& WATER-BASED & & \\
& PROGRAM (4wk) & & \\
& n_6 & &
\end{tabular}

1. For the trunk mobility exercises: To repeat successfully 3 consecutive times $90^{\circ}$ transversal rotations.

2. For the postural stability training objective: To safely maintain the stability on 2 feet while standing on the balance plate for 30s.

3. For the transferring oneself and changing body positions objective: To repeat the exercise 3 consecutive times successfully.

The exercises were selected to match as far as possible the amount of exercise performed and the objective of each exercise between land and aquatic environments. The first part of the exercises was applied for 2 weeks. Progression began at the end of the second week. In the case of any participant who had not successfully achieved the exercise progression, the previous sequence was repeated and a new attempt was tried during the next session. Each exercise was repeated as long as possible within the time available for each section and followed a set sequence (see tables 3 and 4). 
Table 3: Water-Based Program

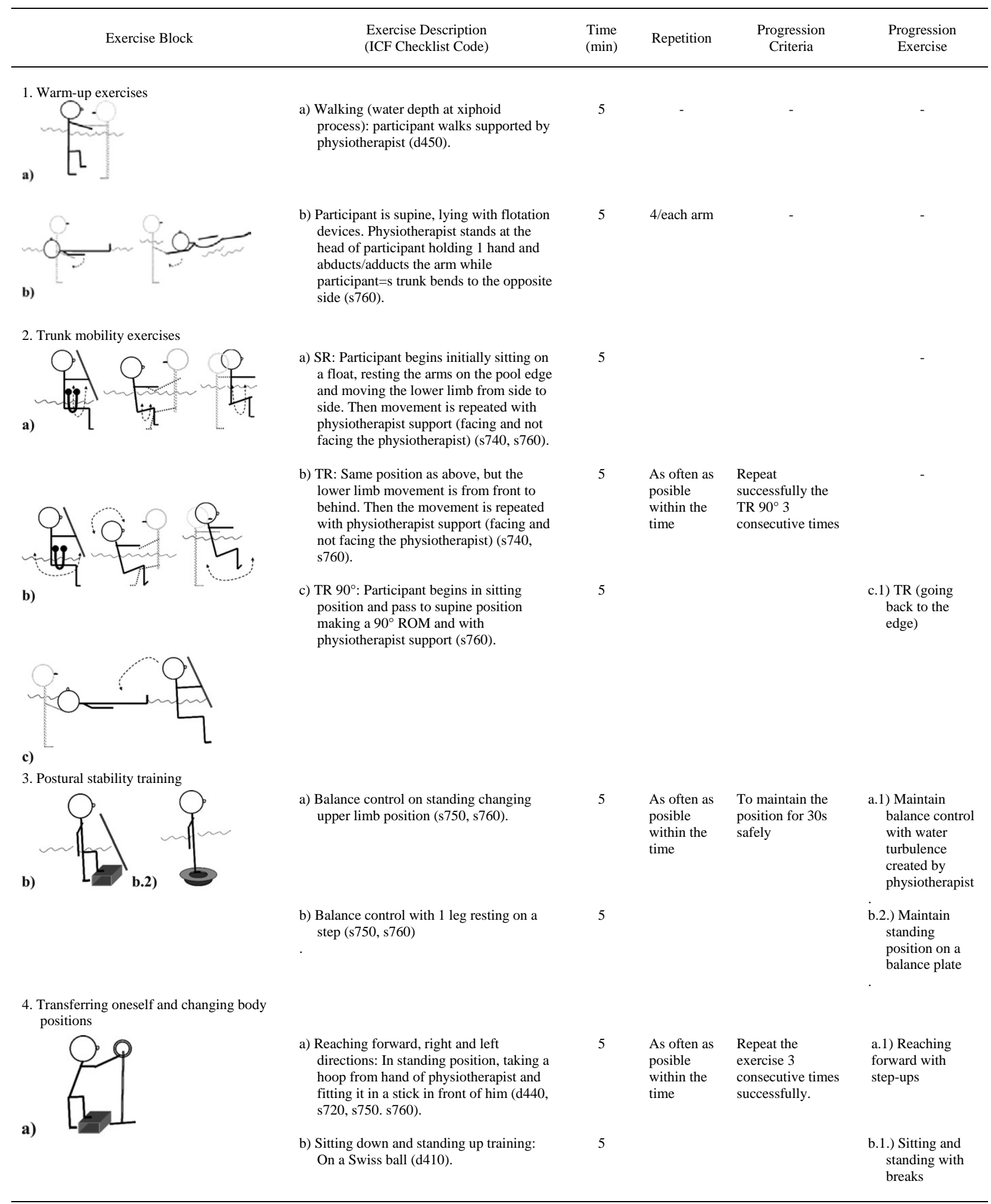

Abbreviations: ICF, International Classification of Functioning, Disability and Health; ROM, range of motion; SR, sagittal rotation: the second point of Halliwick concept, a rotational movement around a sagittal (anteroposterior) axis; TR, transversal rotation: the third point of Halliwick concept, a rotational movement around a latero-lateral axis. 
Table 4: Land-Based Program

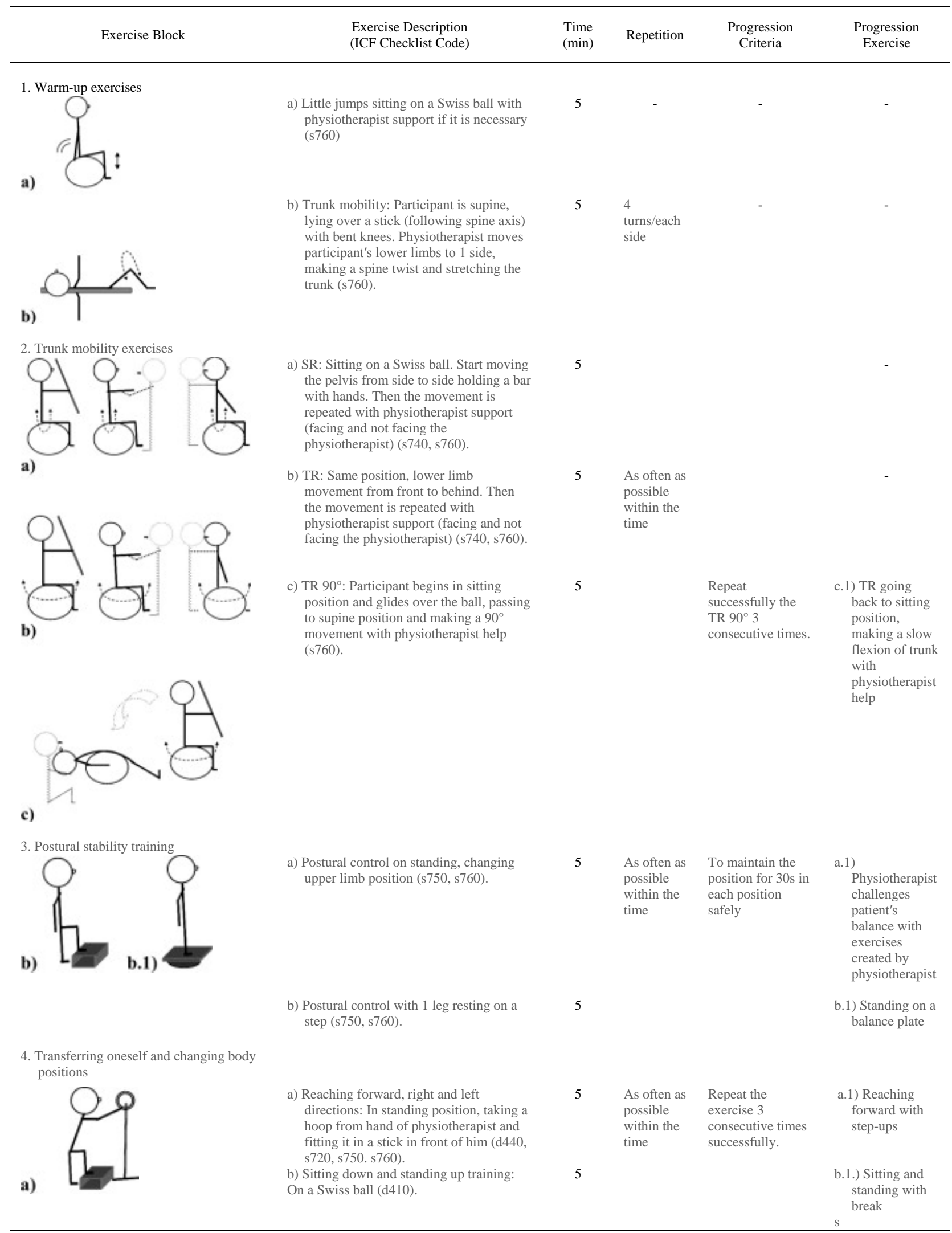

Abbreviations: ICF, International Classification of Functioning, Disability and Health; SR, sagittal rotation: a rotational movement around a sagittal (anteroposterior) axis; TR, transversal rotation: a rotational movement around a latero-lateral axis. 


\section{Analyzed Variables}

The following variables were analyzed:

1. Functional reach distance: The mean functional reaching distance obtained in the 3 trials (the average of both arms), expressed in meters.

2. BBS score

3. Gait

a. Turn time: Obtained from the successive activation of photocells at the end of the corridor (before and after the turn).

b. Velocity: Calculated from the time consumed to cover the straight part of the task, again obtained from the photocells.

c. Cadence (without considering the turn): Number of steps in a given time, assessed from video recording.

d. Step amplitude: Derived from the combination of velocity and cadence.

4. TUG: Time spent to complete the task in seconds.

5. UPDRS score

\section{Data Analysis}

Preliminary analysis revealed that all of the variables were normally distributed (checked by Kolmogorov-Smirnov for 1 sample). To analyze the differences between land and water groups before starting therapy, the Student $t$ test for independent samples was applied for each variable.

To analyze the effect of therapies, an analysis of variance with repeated measures was applied for each variable, and outcomes were interpreted based on main effects and interactions. The within-subject factor was EVALUATION, with 3 levels (Pretest, Posttest, Posttest-2) providing information about outcome changes at the 3 evaluation time points; the between-subject factor was the THERAPY (land or water). A statistically significant outcome of the interaction EVALUATION*THERAPY would prove that water and land protocols had different effects. The SPSS package 14.0 version $^{\mathrm{c}}$ was used for these analyses. A univariate approach was used, and the degrees of freedom were corrected by GreenhouseGeisser coefficients $(\varepsilon)$ in case of sphericity violation. The differences were considered statistically significant with a $P$ value of .05 or less. Throughout this article, any reference to significant difference means statistical significance. Analysis was performed taking only those participants who completed the whole program.

\section{RESULTS}

\section{Characterization of Groups}

Before starting therapy, the groups were similar in all variables analyzed. For gait: turn time $\left(t_{9}=\right.$ 1.063; $P=.346)$; velocity $\left(t_{9}=1.882 ; P=.093\right)$; cadence $\left(t_{9}=1.810 ; P=.104\right)$; step amplitude $\left(t_{9}=\right.$ $1.588 ; P=.147)$. For the other variables: FRT $\left(t_{9}=1.623 ; P=0.139\right)$; $\mathrm{BBS}\left(t_{9}=.846 ; P=.420\right)$; UPDRS total $\left(t_{9}=1.205 ; P=.259\right)$; TUG $\left(t_{9}=1.481 ; P=.173\right)$.

\section{Effect of Therapies}

Variables significantly affected by the protocols. Changes between the evaluation time-points were detected. These were seen in a number of variables directly related to postural stability and functional reaching activities, showing significant improvement (table 5). Therapies significantly improved the functional reach $\left(\mathrm{F}_{2,18}=11.722 ; P=.001\right)$. In addition, because the interaction EVALUATION*THERAPY was not statistically significant for functional reach $\left(\mathrm{F}_{2,18}=2.804 ; P=\right.$ 0.087), both groups (land and aquatic) improved in the same way.

Conversely, a significant interaction EVALUATION*THERAPY was found for the BBS $\left(\mathrm{F}_{2,18}=\right.$ 5.998; $P=.010)$ and for the UPDRS $\left(F_{2,18}=4.012 ; P=.036\right)$, indicating that the effect was different for the group receiving aquatic therapy versus land therapy. We performed a follow-up analysis of variance for each group separately. Only the group receiving aquatic therapy improved the BBS $\left(\mathrm{F}_{2,8}=25.781 ; P\right.$ $=.001)$ and the UPDRS $\left(\mathrm{F}_{2,8}=20.315 ; P=.001\right)$, whereas the land-based therapy group did not significantly change BBS $\left(\mathrm{F}_{2,10}=3.182 ; P=.085\right)$ or UPDRS scores $\left(\mathrm{F}_{2,10}=.965 ; P=.414\right)$.

Variables unaffected by the protocols. For the rest of the tests, the improvement observed in both groups did not reach significance (see table 5). This was detected for the gait variables velocity $\left(\mathrm{F}_{2,18}=\right.$ 2.596; $P=.133 ; \varepsilon=.598)$; step amplitude $\left(\mathrm{F}_{2,18}=1.791 ; P=.195 ; \varepsilon=.555\right)$; turn time $\left(\mathrm{F}_{2,18}=1.705 ; P=\right.$ 
$.210 ; \varepsilon=.523)$; and cadence $\left(\mathrm{F}_{2,18}=2.620 ; P=.100\right)$. The same was observed for the $\mathrm{TUG}\left(\mathrm{F}_{2,18}=2.745\right.$; $P=.124 ; \varepsilon=.589)$. For this group of variables, the interaction EVALUATION*THERAPY was, of course, never significant. Individual values were as follows: turn time $\left(\mathrm{F}_{2,18}=1.213 ; P=.321 ; \varepsilon=.523\right)$; velocity $\left(\mathrm{F}_{2,18}=1.162 ; P=.335 ; \varepsilon=.598\right)$; cadence $\left(\mathrm{F}_{2,18}=1.458 ; P=.259\right)$; step amplitude $\left(\mathrm{F}_{2,18}=\right.$ 2.269; $P=.163 ; \varepsilon=.555) ;$ and TUG $\left(\mathrm{F}_{2,18}=2.317 ; P=.156 ; \varepsilon=.589\right)$.

\section{Program Development}

All participants except 1 completed the program. With regard to the exercise progression regimen, 10 participants achieved progression as intended at the end of the second week of intervention. Only 1 person (a participant in the aquatic therapy group) did not achieve the progression at the first attempt, but did at the following session.

Table 5: Effect of the Therapies

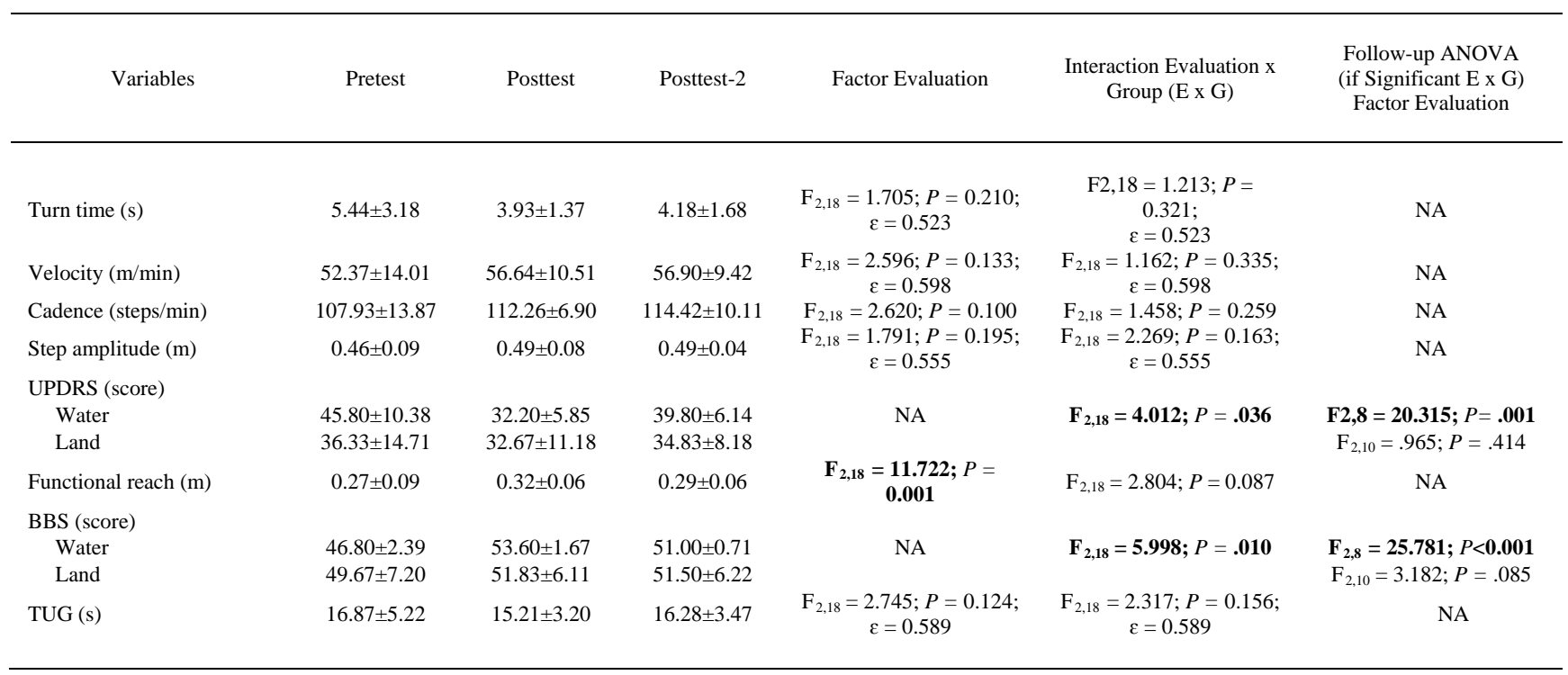

NOTE. Values are mean \pm SD or as otherwise indicated. The table represents the effect of Factor Evaluation along the whole protocol. In case of significant interaction (E $x$ G), which means the effect is different for both groups, a follow-up ANOVA was performed, 1 for each group. Analysis shows functional reach was improved. Improvement was the same for both groups (lack of significant interaction E x G). Significant E x G was seen, however, for BBS and UPDRS, showing both groups were affected differently. The follow-up ANOVA showed that only the water group improved in these variables. Analysis in the rest of the variables showed lack of significant Factor Evaluation, or E x $G$ interaction, proving lack of effect on them. Values for each variable are displayed at the beginning of the program (Pretest), after finishing the program (Posttest), and after follow-up (Posttest-2). In the case of lack of significant interaction Evaluation x Group, values are shown for both groups pooled, given the effect was the same. Split values (BBS and UPDRS) are based on significant interaction Evaluation x Group, proving different behavior of both groups. Significant main effects or interactions are indicated in bold. Abbreviations: ANOVA, analysis of variance; NA, not applicable.

\section{DISCUSSION}

In this pilot study, we presented and compared 2 different protocols of physiotherapy (land- and water-based therapy). Additionally, this study showed that both land- and water-based protocols could be useful for affected aspects of balance in individuals with PD.

These preliminary positive results suggest that the intervention protocols seem suitable for PD. Importantly, the intervention phases and criteria to pass from 1 phase to the next seem appropriate because they promote improvements and were very well tolerated. Further, only one subject did not achieve the progression criteria on schedule; therefore, it is possible that we set progression criteria that were too easily achieved. While diminish the clinical effect. This should be borne in mind in the design of any larger study.

Both physiotherapy protocols used were based on some approaches and techniques that are commonly used in clinical practice for neurologic patients, including those with PD. We chose primarily 3 procedures - trunk mobility exercises (rotations), balance training, and task-oriented exercises-all of which focused on postural stability problems.

Our methodological approach included matching both protocols as closely as possible. We intended to reproduce the basic rotational exercises of the Halliwick method, ${ }^{28,29}$ and the instability felt by the 
participants in the water was recreated by using a Swiss ball in the land exercises. In both protocols, the aims were to stimulate trunk mobility in different planes, inducing participants to move their center of gravity away from its base of support, training for upright control and for balancing reactions in unusual conditions.

Buoyancy and hydrostatic pressure offered by water promote body support and reduce the velocity of falls. The water-based protocol appears to have better results compared with those for the land-based therapy, because it promoted changes not only in reaching but also in the BBS, which might improve learning by providing more time to perform compensatory motor programs. On the other hand, warm water may have a potential therapeutic effect on rigidity, ${ }^{29}$ a cardinal sign in PD. Further studies should seek to confirm that parkinsonian rigidity, which has a central origin, ${ }^{30}$ is relieved by peripheral stimulation such as warmth.

We should also consider, however, the unavoidable differences between protocols. For instance, reaching forward has different implications on land and in water. On land, the control of the projection of the center of gravity with respect to the support base area is very demanding for the posterior muscles of the body, which is less demanding in the case of being in shallow water, given the action of buoyancy. Buoyancy also provides extra support for the participant to perform the task, which could be 1 of the reasons why we obtained better results with the water protocol. The reduced fear of falling in water is another important aspect to be considered.

We matched both aquatic and land programmes in terms of the amount of exercise performed. Some other experimental variables were initially considered-for example, heart rate or perceived rate of exertion. However, in the case of the aquatic protocol, we understand that the perceived rate of exertion will be very different, with "novelty" being a factor that could alter perceived effort. This could bias the outcomes, given the possibility of extra motivation or higher expectancy in the water group.

With respect to heart rate, a confounding factor would be that heart rate changes can occur after warm-water immersion, although these can be corrected for. We reasoned, however, that heart rate might be a more useful parameter if we were focusing our objective on some physiologic capacities, such as maximum oxygen consumption or anaerobic threshold. However, these are more related to bioenergetics than to measurement of, or improvement in, deficient motor control, which is the hallmark in PD.

Progressive criteria (an important and novel aspect of our work) were established, based on the objective targeted. On that basis, we selected criteria for the different abilities to be achieved:

1. Trunk mobility: Participants were required to complete successfully the transversal rotation $90^{\circ}$ three consecutive times. Motor control of postural responses to instability are deficient in PD, and axial rigidity is a cardinal sign in the disease, which leads participants to be impaired on functional mobility in the sagittal and transverse planes. The exercises were organized from movements of smaller amplitude to larger amplitude, so that reaching $90^{\circ}$ was a functional target (from less demanding to more demanding).

2. Postural stability training: Postural instability is the sign in PD with the greatest impact on quality of life because it is related to gait impairment and risk of falling. ${ }^{31}$ For this reason we included training elements aimed at gaining abilities related to postural stability and proposed exercises focused on postural adjustments made to compensate for the instability created by the tasks. Water unsteadiness and turbulence created by the physiotherapist, or unbalancing actions of the physiotherapist on the participant, were the latest training elements proposed based on being much more demanding than those at the beginning. These were aimed to gain control on the postural mechanism responsible for improving muscle functioning in stability-demanding tasks. Unsteadiness during land exercises was recreated by elements such as the Swiss ball.

3. Transferring oneself and changing body positions: Reaching forward, reaching to the right and left, sitting down, and standing up were criteria selected because those abilities are impaired in PD.

One basic element that must be unequivocally taken into account when considering the progression criteria is the ability level of the participants at the beginning of the protocol. The average group disease development was about 2.5, and a number of participants were in stage 3 of the disease based on the Hoehn and Yahr Scale, a phase at which postural instability is manifest. The program presented in the study must of course be adapted to the different ability levels of the individuals.

Trying to identify why the stability of the participants improved with both aquatic- and land-based protocols may be misleading if not supported by neurophysiologic data. Perhaps the most parsimonious explanation would be that the improvement was due to a more efficient activation of the postural muscles; this can be further confirmed in future studies. Learning new strategies in order to gain stability is also an option, mainly if participants are conscious of postural adjustments that provide benefit. Of course, from a scientific viewpoint, the role of placebo must not be ruled out in any open-label study. The placebo effect 
could also be the reason why a more innovative approach (water based), compared with a traditional one (land based), has a better effect. All of these elements should be controlled in the future.

These considerations aside, our pilot study results seem to agree reasonably with those of other studies showing positive effects of physiotherapy in PD. The data from the study of Viliani et al1 demonstrate that muscle and range limitations contribute to reduced upright capacity, and that recovery of trunk and pelvis mobility was a useful tool to prevent the worst effects of the disability. Van Vaerenbergh et $\mathrm{al}^{32}$ showed that rotational stimulation might provide nonpharmacologic relief from freezing for individuals with PD. Additionally, a number of studies have indicated that physiotherapy improves the UPDRS score and, in particular, the motor and daily life activity subscales of the UPDRS. ${ }^{33}$

Specific studies using exclusively water-based therapy for people with PD are scarce. We have only found 2 articles $^{3,35}$ that included aquatic activities as part of the protocol. Pellecchia et $\mathrm{al}^{3}$ observed improvements in the UPDRS score (activities of daily living section) and gait after a 20-week program, effects that lasted 3 months. Brefel-Courbon et $\mathrm{al}^{35}$ evaluated the effectiveness of spa therapy in the management of individuals in a pilot study with PD, finding improvements in the quality-of-life questionnaire and part IV of the UPDRS. The duration of any intervention is another point to be considered. If a protocol is useful, it must be included in the everyday therapeutic approach for PD. However, within any program under experimental conditions, limits and follow-up periods without treatment must be defined. For example, positive effects have been described in a study ${ }^{35}$ with a shortterm intervention, and it is possible that the effects obtained by other researchers during larger periods of intervention3 might have been achieved in a shorter period of time; this is unknown because often designs do not include intermediate evaluation.

\section{Study Limitations}

Pilot studies are justified when providing information to guide future, larger trials. There are several methodological considerations derived from this study that should be considered in that respect. One is to control the effect of expectancy. Controlling the role of placebo is a key element in scientific designs that must be included in larger trials, ${ }^{36}$ since this can be a clear physiologic mechanism that might explain the results of our aquatic therapy pilot work. However, controlling the effect of placebo becomes difficult in physiotherapy. A group receiving an ineffective protocol is a possibility, but ethical considerations then arise. "Dose dependency" could be considered, given that the extent of a therapeutic effect will depend on dose-in this case, for example, more or longer sessions should have a better effect than fewer sessions. A design with a longer follow-up period is also useful to control placebo-related effects, because they are known to fade away faster than real effects. ${ }^{37}$ Clearly open-label studies such as we report here have advantages as a first approach, but single-blind or double-blind studies are needed. A larger trial should include evaluators totally unaware of the groups they are evaluating, and the use of "different doses" will keep participants unaware of the group they belong to.

The drug state of the participants is also a basic element to control in the future. We have designed this pilot study to evaluate participants while OFF drugs. It provides advantages in terms of stability of the measured signs, since PD symptoms may fluctuate in relation to drug intake. However, the effect obtained might be dependent on drug state, and evaluation during ON and OFF periods is needed. This approach has been proposed in some other therapeutic options. ${ }^{38-40}$

Finally, we have focused our work on the axial problems of the disease, but other effects of the aquatic program presented here may include a greater impact on some other PD symptoms such as limb rigidity. Larger studies should investigate a wider range of possible improvements.

\section{CONCLUSIONS}

This pilot study shows the impact of an aquatic physiotherapy protocol on postural stability in PD and provides the basis for future research with larger trials. Aquatic therapy in this protocol seems to be more effective than land-based protocols, and some of the methodological aspects detailed here should be considered when designing larger controlled trials. Such trials should be aimed not only at reinforcing the view that physiotherapy is useful as supportive therapy in PD (and doing so in a scientifically rigorous manner) but also at investigating the extent to which aquatic protocols can provide further benefit, complementing the benefits that can be derived from the more accessible land-based protocols. 
Acknowledgment: We thank Kenneth Grieve, $\mathrm{PhD}$, for his corrections and critical reading of the manuscript.

\section{References}

1. Viliani T, Pasquetti P, Magnolfi S, et al. Effects of physical training on straightening-up processes in patients with Parkinson's disease. Disabil Rehabil 1999;21:68-73.

2. Behrman AL, Cauraugh JH, Light KE. Practice as an intervention to improve speeded motor performance and motor learning in Parkinson’s disease. J Neurol Sci 2000;174:127-36.

3. Pellecchia MT, Grasso A, Biancardi LG, Squillante M, Bonavita V, Barone P. Physical therapy in Parkinson's disease: an open long-term rehabilitation trial. J Neurol 2004;251:595-8.

4. Kwakkel G, de Goede CJ, van Wegen EE. Impact of physical therapy for Parkinson's disease: a critical review of the literature. Parkinsonism Relat Disord 2007;13:S478-87.

5. Deane KHO, Ellis-Hill C, Jones D, Whurr R. Systematic review of paramedical therapies for Parkinson's disease. Mov Disord 2002; 17:984-91.

6. Formisano R, Pratesi L, Modarelli FT, Bonifati V, Meco G. Rehabilitation and Parkinson’s disease. Scand J Rehabil Med 1992;24:157-60.

7. Miyai I, Fujimoto Y, Ueda Y, et al. Treadmill training with body weight support: its effect on Parkinson's disease. Arch Phys Med Rehabil 2000;81:849-52.

8. Ellis T, de Goede CJ, Feldman RG, Wolters EC, Kwakkel G, Wagenaar RC. Efficacy of a physical therapy program in patients with Parkinson's disease: a randomized controlled trial. Arch Phys Med Rehabil 2005;86:626-32.

9. Peppe A, Chiavalon C, Pasqualetti P, Crovato D, Caltagirone C. Does gait analysis quantify motor rehabilitation efficacy in Parkinson's disease patients? Gait Posture 2007;26:452-62.

10. Nieuwboer A, De Weerdt W, Dom R, Truyen M, Janssens L, Kamsma Y. The effect of a home physiotherapy program for persons with Parkinson's disease. J Rehabil Med 2001;33:266-72.

11. Katsura Y, Yoshikawa T, Ueda SY, et al. Effects of aquatic exercise training using water-resistance equipment in elderly. Eur J Appl Physiol 2010;108:957-64.

12. Devereux K, Robertson D, Briffa NK. Effects of a water-based program on women 65 years and over: a randomised controlled trial. Aust J Physiother 2005;51:102-8.

13. Arnold CM, Busch AJ, Schachter CL, Harrison EL, Olszynski WP. A randomized clinical trial of aquatic versus land exercise to improve balance, function, and quality of life in older women with osteoporosis. Physiother Can 2008;60:296-306.

14. Suomi R, Koceja DM. Postural sway characteristics in women with lower extremity arthritis before and after an aquatic exercise intervention. Arch Phys Med Rehabil 2000;81:780-5.

15. Gabilan YP, Perracini MR, Munhoz MS, Gananç FF. Aquatic physiotherapy for vestibular rehabilitation in patients with unilateral vestibular hypofunction: exploratory prospective study. J Vestib Res 2008;18:139-46.

16. Kelly M, Darrah J. Aquatic exercise for children with cerebral palsy. Dev Med Child Neurol 2005;47:838-42.

17. Franchimont P, Juchmest J, Lecomte J. Hydrotherapy-mechanisms and indications. Pharmacol Ther 1983;20:79-93.

18. Kesiktas N, Paker N, Erdogan N, Gulsen G, Biçki D, Yilmaz H. The use of hydrotherapy for the management of spasticity. Neurorehabil Neural Repair 2004;18:268-72.

19. Koury JM. Aquatic therapy programming-guidelines for orthopaedic rehabilitation. Champaign: Human Kinetics; 1996.

20. Kovács I, Bender T. The therapeutic effects of Cserkeszölö thermal water in osteoarthritis of the knee: a double blind, controlled, follow-up study. Rheumatol Int 2002;21:218-21.

21. Kesiktas N, Paker N, Erdogan N, Gülsen G, Biçki D, Yilmaz H. The use of hydrotherapy for the management of spasticity. Neurorehabil Neural Repair 2004;18:268-73.

22. Morris ME, Huxham F, McGinley J, Dodd K, Iansek R. The biomechanics and motor control of gait in Parkinson disease. Clin Biomech 2001a;16:459-70.

23. O’Sullivan JD, Said CM, Dillon LC, Hoffman M, Hughes AJ. Gait analysis in patients with Parkinson's disease and motor fluctuations: influence of levodopa and comparison with other measures of motor function. Mov Disord 1998;13:900-6.

24. Mesure S, Azulay JP, Pouget J, Amblard B. Strategies of segmental stabilization during gait in Parkinson’s disease. Exp Brain Res 1999;129:573-81. 
25. Duncan PW, Weiner DK, Chandler J, Studenski S. Functional reach: a new clinical measure of balance. J Gerontol 1990;45:M192-7.

26. Berg KO, Wood-Dauphinee SL, Williams JI, Maki B. Measuring balance in the elderly: validation of an instrument. Can J Public Health 1992;83:S7-11.

27. Podsiadlo D, Richardson S. The Timed 'Up \& Go': a test of basic functional mobility for frail elderly persons. J Am Geriatr Soc 1991;39:142-8.

28. McMillan J. The role of water in rehabilitation. Fysioterapeuten 1978;45:87-90.

29. Brody LT, Geigle PR. Aquatic exercise for rehabilitation and training. Champaign: Human Kinetics; 2009.

30. Delwaide PJ, Pepin JL, Maertens de Noordhout A. Parkinsonian rigidity: clinical and physiopathologic aspects. Rev Neurol (Paris) 1990;146:548-54.

31. Jankovic J. Parkinson's disease: clinical features and diagnosis. J Neurol Neurosurg Psychiatry 2008;79:368-76.

32. Van Vaerenbergh J, Vranken R, Baro F. The influence of rotational exercises on freezing in Parkinson's disease. Funct Neurol 2003;18:11-6.

33. Dam M, Tonin P, Casson S, et al. Effects of conventional and sensory-enhanced physiotherapy on disability of Parkinson's disease patients. Adv Neurol 1996;69:551-5.

34. Schenkman M, Cutson TM, Kuchibhatla M, et al. Exercise to improve spinal flexibility and function for people with Parkinson's disease: a randomized, controlled trial. J Am Geriatr Soc 1998;46:120716.

35. Brefel-Courbon C, Desboeuf K, Thalamas C, et al. Clinical and economic analysis of spa therapy in Parkinson's disease. Mov Disord 2003;18:578-84.

36. Arias P, Vivas J, Grieve KL, Cudeiro J. Double-blind, randomized, placebo controlled trial on the effect of 10 days low frequency rTMS over the vertex on sleep in Parkinson's disease. Sleep Med 2010;11:759-65.

37. Oken BS. Placebo effects: clinical aspects and neurobiology. Brain 2008;131:2812-23.

38. Lomarev MP, Kanchana S, Bara-Jimenez W, Iyer M, Wassermann EM, Hallett M. Placebo-controlled study of rTMS for the treatment of Parkinson's disease. Mov Disord 2006;21:325-31.

39. Arias P, Chouza M, Vivas J, Cudeiro J. Effect of whole body vibration in Parkinson's disease: a controlled study. Mov Disord 2009;24:891-8.

40. Arias P, Vivas J, Grieve KL, Cudeiro J. Controlled trial on the effect of 10 days low-frequency repetitive transcranial magnetic stimulation (rTMS) on motor signs in Parkinson's disease. Mov Disord 2010;25:1830-8.

\section{Suppliers}

a. Holtain Ltd. Crosswell, Crymych, Pembs., SA41 3UF, UK.

b. JVC Kenwood Holdings. Available at: http://www.jk-holdings.com/en/index.html.

c. SPSS Inc, 233 S Wacker Dr, 11th Fl, Chicago, IL 60606. 